\title{
Carbohydrate Metabolism during Sporulation in Spheroplasts of Yeast, Saccharomyces cerevisiae
}

\author{
Shigeyoshi Katohda, Hiroshi Ito, Hideki TaKahashi \\ and Hiroshi KIKUCHI \\ Laboratory of Applied Microbiology, Faculty of Agriculture, \\ Yamagata University, Tsuruoka, Yamagata 997, Japan
}

Received August 10, 1987

\begin{abstract}
Carbohydrate metabolism during sporulation in spheroplasts of Saccharomyces cerevisiae was examined to detect changes in the synthesis and degradation of cellular materials. The cellular materials in spheroplasts were fractionated into extraspore material, spore-cytoplasm and spore walls. Glycogen in the extraspore material was synthesized early in development and was degraded prior to spore maturation. Trehalose, glycogen and mannan in the spore-cytoplasm were all synthesized during spore formation. Glucan, as a major component of the spore walls, was synthesized during spore formation. A glucosamine polymer, as a component of the outer layers of the spore walls, was synthesized immediately after incubation in the sporulation medium. Mannan synthesis, as a minor component in the spore walls, was completed prior to spore maturation.
\end{abstract}

There have been many studies on the carbohydrate metabolism during ascospore development in Saccharomyces cerevisiae. $^{1 \sim 9)}$ However, since asci of the yeast were used in these previous studies, it is possible that some of the carbohydrate was derived from preexisting material in the original ascus wall. Since the ascus wall is not involved, it is expected to be advantageous for studies on carbohydrate metabolism if sporulation in spheroplasts can be induced with high synchrony and frequency. The yeast strain, FE-1, used in the present study is a natural variant showing remarkable sporulation ability ${ }^{10,11)}$ with high synchrony and frequency under osmotic conditions in spheroplasts. ${ }^{12)}$ Using the technique of sporulation in spheroplasts, it has been possible to determine the distribution of cellular carbohydrates during sporulation. ${ }^{13)}$ Cellular materials in spheroplasts were fractionated into extraspore material, spore-cytoplasm and spore walls. ${ }^{14)}$

In the present study, we examined changes in carbohydrate metabolism in these three fractions during sporulation in spheroplasts.

\section{MATERIALS AND METHODS}

Organism and culture conditions. A diploid homothallic Saccharomyces cerevisiae strain, FE-1, orginally obtained from Dr. Sando, was used in this work. The procedures for growth, spheroplast formation and sporulation were described previously. ${ }^{13)}$ For sporulation in spheroplasts, the culture was carried out at $30^{\circ} \mathrm{C}$ on a rotary shaker in $100 \mathrm{ml}$ of $60 \mathrm{~mm}$ potassium acetate containing $0.75 \mathrm{M}$ sorbitol in a $500-\mathrm{ml}$ flask at a density of $3 \times 10^{7}$ spheroplast cells $/ \mathrm{ml}$. Samples were taken at various intervals and used for determination of the chemical composition or for fractionation of cellular materials.

Microscopic observation. The extent of sporulation was expressed as the percentage of sporulated cells, determined by examining morphologically completed asci under a microscope. Also, observations using the fluorescent dye, Brightener 28 (Sigma), were made as described previously. ${ }^{14\}}$

Fractionation of extraspore material, spore-cytoplasm and spore walls from spore-induced spheroplasts. After spheroplasts had been incubated in the sporulation medium, they were collected by centrifugation $(2000 \times g$, $5 \mathrm{~min}$ ) and then washed 3 times with $0.8 \mathrm{M} \mathrm{KCl}$. The sedimented spheroplasts were disrupted by suspending in distilled water. The disrupted suspension was washed 3 times with distilled water by centrifugation. The supernatants obtained were combined and defined as the 
extraspore material. The extraspore material was heated in a boiling water bath for $5 \mathrm{~min}$ and then subjected to carbohydrate analysis after dialysis against water and then lyophilization. About $1 \mathrm{~g}$ (wet weight) of the precipitated pellet, which was composed of ascospores, was resuspended in $20 \mathrm{ml}$ of distilled water, mixed with $30 \mathrm{~g}$ of glass beads and then shaken with a Braun cell homogenizer. The cell homogenate was centrifuged $(2000 \times g, 5 \mathrm{~min})$. The pellet, defined as the spore walls, was washed 10 times with distilled water by centrifugation. The combined supernatants were defined as the spore-cytoplasm, which was subjected to chemical analysis either directly or after lyophilization. The spore walls were divided into alkali-soluble and -insoluble fractions by treatment with $1 \mathrm{~N} \mathrm{KOH}$ at $20^{\circ} \mathrm{C}$ for $24 \mathrm{hr}$ as described previously. ${ }^{(3)}$ The soluble and insoluble fractions were neutralized, dialyzed against water and then lyophilized.

Analytical methods. The carbohydrate in the extraspore material was almost all glycogen. The spore-cytoplasm contained carbohydrates such as glycogen, trehalose and mannan. Polysaccharides, fractionated by chromatography on Bio-Gel P-4, were collected, concentrated by evaporation in vacuo and then used as the glycogen sample. The latter was analyzed by the amyloglycosidase digestion method as described previously. ${ }^{14)}$ To determine the amount of trehalose, the disaccharide fractions obtained on Bio-Gel P-4 chromatography were analyzed directly with phenol-sulfuric acid reagent. To determine the amount of mannan in the spore-cytoplasm, the polysaccharide fraction was evaluated as total mannose. The alkali-soluble and -insoluble fractions of the spore walls were analyzed by gas liquid chromatography for sugar content determinations. The glucose, mannose, glucosamine and protein determinations were carried out as described previously. ${ }^{13}$ )

Methylation analysis. The alkali-soluble fraction of the spore walls was methylated by the Hakomori method, ${ }^{15}$ ) and then analysis was carried out as described previously. ${ }^{16)}$

\section{RESULTS}

\section{Microscopic observation}

We were able to demonstrate that chitin is the intrinsic entity for vegatative yeast cells, constituting the annular structure of the bud scar region, ${ }^{17)}$ and for germinated yeast spores, constituting the outer layer of the spore wall, ${ }^{14)}$ and that it could be stained with a Brightener solution $\left(5 \times 10^{4}\right.$ dilution $)$. Figure 1 shows phase-contrast and fluorescence micrographs taken during sporulation in sphero- plasts of Saccharomyces cerevisiae strain FE1. The phase-contrast micrographs of the spheroplast preparations show spherical spheroplasts between 0 - and 12-hr incubation (Figs. $1 \mathrm{~A} \sim \mathrm{D}$ ). The spheroplasts enlarged cell size during the first 4-hr incubation (Fig. 1B). Non-refractile prespores appeared in spheroplast after 8-hr incubation (Fig. 1C). Figures ID and $\mathrm{E}$ show spherical spores in spheroplasts after 12-hr incubation on phasecontrast and fluorescence microscopy, respectively. The spores show fluorescence (Fig. 1E). The appearance of the spores on fluorescence microscopy after 12-hr incubation indicated that the fluorescent substance was located on the surface of the spores. Figure $1 \mathrm{~F}$ shows typical ascospores after 16-hr incubation on phase-contrast microscopy. The spores could not be stained with the fluorescent dye (data not shown). After 16-hr incubation, the disappearance of the spores, as seen on fluorescence microscopy, indicated that the fluorescent substance was covered with or converted to other substances during spore maturation.

\section{Kinetics of sporulation and chemical com- positions}

The sporulation kinetics and change in dry weight of spores are shown in Fig. 2A. The percentage of asci rose from 0 to $80 \%$ between 6- and 16-hr incubation, and had reached $92 \%$ by $20 \mathrm{hr}$. Spore weight increased during the first 5-hr incubation, after which it decreased during the next 5-hr incubation with a subsequent increase with the progress of sporulation. Figure 2B shows cellular carbohydrates during sporulation. Glucose increased from about 20 to $200 \mu \mathrm{g} / 10^{8}$ spheroplasts between 0 and $4 \mathrm{hr}$. Between 4 and $8 \mathrm{hr}$, when $3 \%$ asci had appeared, glucose decreased to $130 \mu \mathrm{g} / 10^{8}$ spheroplasts. After $8 \mathrm{hr}$, the glucose content again rose, reaching a level of $180 \mu \mathrm{g} / 10^{8}$ spheroplasts by $16 \mathrm{hr}$. During spore formation ( 8 to $16 \mathrm{hr}$ ), mannose increased from about 20 to $60 \mu \mathrm{g} / 10^{8}$ spheroplasts. A small amount of glucosamine appeared in the spheroplasts, increasing slowly during spore formation.

The spore and extraspore materials were 

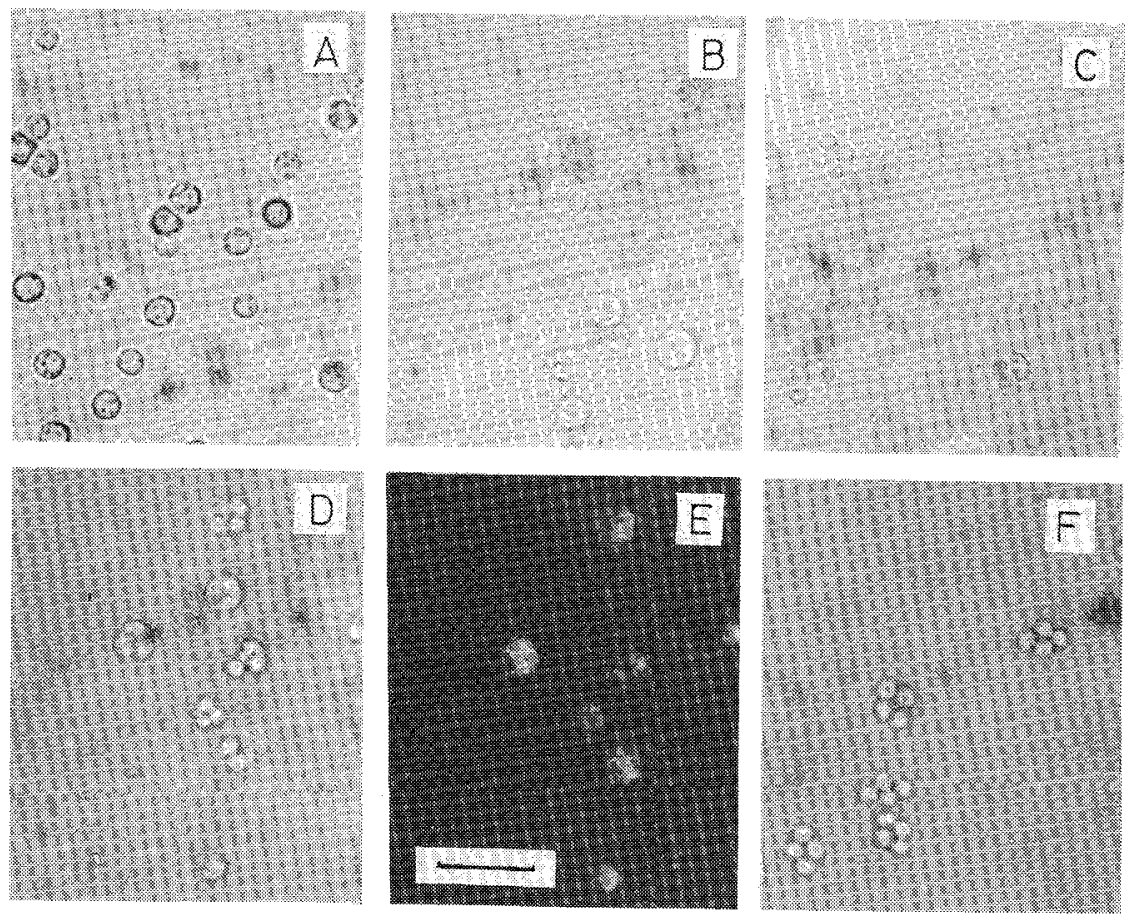

FIG. 1. Micrographs during Sporulation in Spheroplasts of $S$. cerevisiae.

Spheroplasts (A) incubated in the sporulation medium after $4(\mathrm{~B}), 8(\mathrm{C}), 12$ (D and E) and $16 \mathrm{hr}(\mathrm{F})$, observed under a phase-contrast $(A, B, C, D$, and F) or a fluorescence (E) microscope. Bar, $10 \mu \mathrm{m}$.

fractionated by centrifugation. Glycogen in the extraspore material increased six-fold, from 25 to $150 \mu \mathrm{g} / 10^{8}$ spheroplasts, between 0 and $4 \mathrm{hr}$, and then decreased to $10 \mu \mathrm{g} / 10^{8}$ spheroplasts at $12 \mathrm{hr}$ (Fig. 3A). The period of glycogen disappearance coincided with the appearance of prespores and with the decrease in glucose show in Fig. 2B. After 16-hr incubation, sugars could no longer be detected in the extraspore material. Figure 3B shows spore carbohydrates during sporulation. The accumulation of glucose and glucosamine began immediately after incubation of spheroplasts in the sporulation medium. Glucosamine accumulation was completed prior to the appearance of mature spores and continued throughout the remaining incubation period, whereas glucose accumulation was completed within the period of spore formation. Protein accumulation was similar to glucosamine accumulation. Mannose accumulation coincided with the appearance of pre-

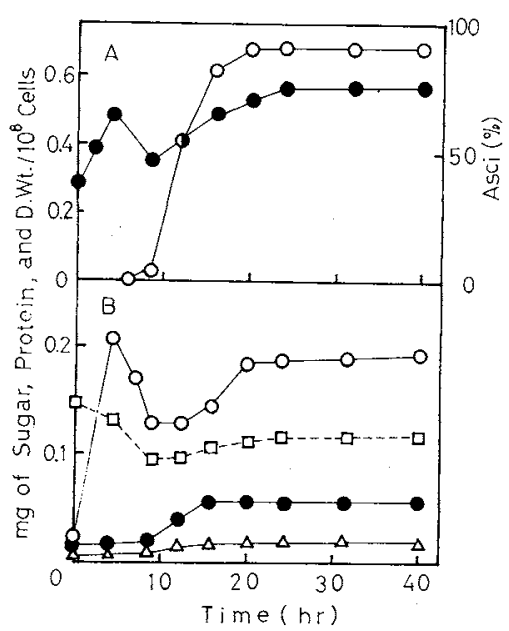

Fig. 2. The Kinetics and Changes in Cellular Composition during Sporulation in Spheroplasts.

At intervals, samples were withdrawn for the determination of the percentage of asci and observation by phasecontrast microscopy. For the subsequent determination of both dry weight of and chemical composition of sporeinduced spheroplasts, samples were collected by centrifugation, dialyzed against water and then lyophilized.

A: $O$, percentage of asci; $O$, dry weight of spores.

B: $\bigcirc$, glucose; $\bigcirc$, mannose; $\triangle$, glucosamine; $\square$, protein. 


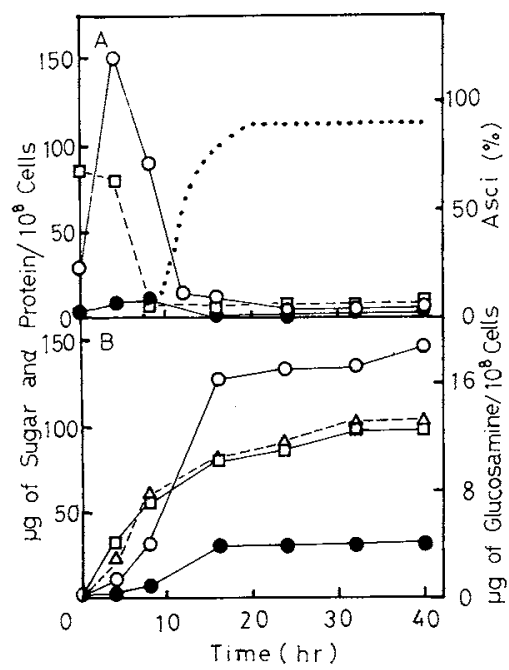

FIG. 3. Changes in Sugar and Protein in Extraspore Material and Spores during Sporulation in Spheroplasts.

Spheroplasts samples were fractionated as described under Materials and Methods.

A: The contents of glycogen $(O)$, mannan $(O)$ and protein $(\square)$ in the extraspore material are shown here. The dotted line shows the sporulation kinetics, as determined in Fig. 1.

B: The contents of glucose $(O)$, mannose $(\mathbf{O})$, glucosamine $(\triangle)$ and protein $(\square)$ in the cellular material of spores are shown here.

spores and was completed prior to spore maturation.

Carbohydrate analysis of the spore walls and spore-cytoplasm

To determine the amounts of storage carbohydrates, we fractionated the sporecytoplasm carbohydrates into trehalose and glycogen by chromatography on Bio-Gel P-4 as described previously. ${ }^{14)}$ Trehalose increased between 4 and $8 \mathrm{hr}$, remained constant between 8 and $12 \mathrm{hr}$ (when spores were forming) and then rose again between 12 and $16 \mathrm{hr}$ (Fig. 4A). The accumulation of glycogen and mannan occurred coincidently with spore formation. Of the spore-cytoplasm carbohydrates, trehalose was most abundant, followed by mannan and glycogen. Figure 4B shows spore wall carbohydrates during sporulation. Glucose synthesis began prior to the appearance of prespores and was completed within the period of spore formation. Man-

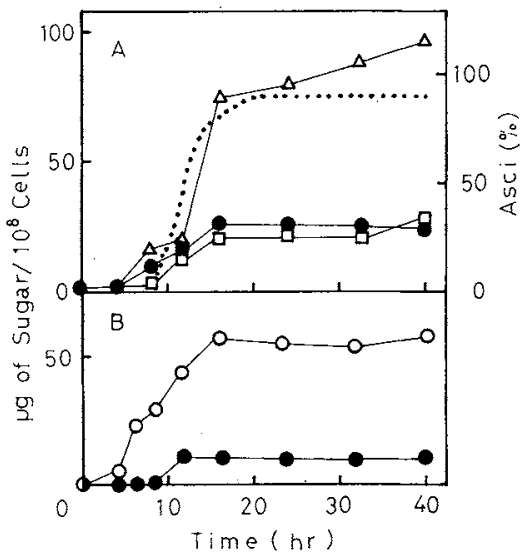

FIG. 4. Changes in Sugar Compositions of the Sporecytoplasm and Spore Walls during Sporulation in Spheroplasts.

Spores were harvested and fractionated as described under Materials and Methods.

A: The contents of trehalose $(\triangle)$, mannan $(\mathbf{O})$ and glycogen $(\square])$ in the spore-cytoplasm are shown here. The dotted line represents the sporulation kinetics, as determined in Fig. 1.

$\mathrm{B}$ : The contents of glucose $(O)$ and mannose $(O)$ in the spore walls are shown here.

nan synthesis was completed prior to the spore formation, but the levels were very low. In the mature spore walls, glucose exceeded, by about 6-fold, mannose.

The wall materials were fractionated by extraction with $1 \mathrm{~N} \mathrm{KOH}$ for $24 \mathrm{hr}$ at $20^{\circ} \mathrm{C}$. Since the alkali-soluble fractions showed no reducing power, it appeared that only large moleculars were released on the extraction. The amounts of carbohydrates (glucose, mannose and glucosamine) in the alkali-soluble and -insoluble fractions are shown in Fig. 5. In the alkali-soluble fraction, glucose increased from 13 to $42 \mu \mathrm{g} / 10^{8}$ spheroplasts, and then decreased to $38 \mu \mathrm{g} / 10^{8}$ spheroplasts at $24 \mathrm{hr}$ (Fig. 5A). Moreover, to clarify the structural change in the glucose polymer contained to the alkali-soluble fraction during sporulation ( 8 to $24 \mathrm{hr}$ ), preparations were subjected to methylation analysis (Table I). The results showed that the alkali-soluble fraction of spore walls contained mainly the 1,3-glucosidic linkages, and little of the 1,6- and 1,4-glucosidic link- 


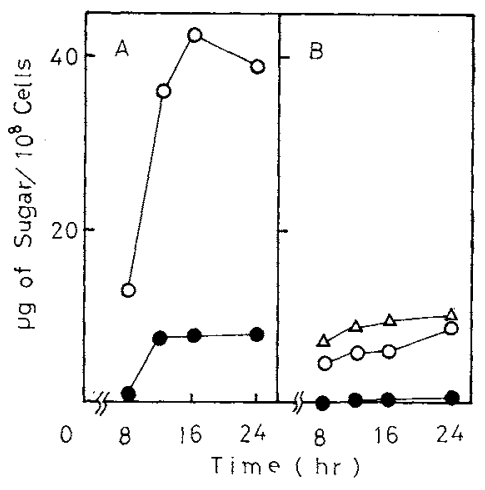

FIG. 5. Changes in Sugar Compositions of the Alkalisoluble and -insoluble Fractions of the Spore Walls during Sporulation in Spheroplasts.

A: The contents of glucose (O) and mannose (O) in the alkali-soluble fraction of the spore walls are shown here. B: The contents of glucose $(O)$, mannose $(\bigcirc)$ and glucosamine $(\triangle)$ in the alkali-insoluble fraction of the spore walls are shown here.

Table 1. Methanolysis Products of the Methylated Alkali-soluble Fraction OF SPORE WALLS

Molar ratios as glucose

Methyl glucose Incubation time in sporulation medium (hr)

\begin{tabular}{lrrrr} 
& 8 & 12 & 16 & 24 \\
\hline $2,3,4,6$-Tetra- & 2.5 & 1.7 & 1.8 & 2.9 \\
$2,3,4$-Tri- & 9.5 & 9.4 & 13.7 & 14.7 \\
$2,4,6$-Tri- & 68.8 & 71.1 & 59.3 & 62.8 \\
$2,3,6$-Tri- & 15.2 & 14.2 & 21.2 & 15.7 \\
2,4 -Di- & 4.0 & 3.6 & 4.0 & 3.9 \\
\hline
\end{tabular}

ages, during sporulation. The contents of 1,4- and 1,6-linked residues increased between 16 and $24 \mathrm{hr}$. The contents of 1,6-linked residues remained constant between 16 and $24 \mathrm{hr}$. The contents of 1,4-linked residues decreased again between 16 and $24 \mathrm{hr}$. Methylated mannosides were not detected on analysis by gas liquid chromatography with $5 \%$ OV-17 on Chromosorb W AW. The spore wall-associated mannose could be completely released as mannan on alkali-extraction (Figs. 4B and 5A). Mannan accumulation coincided with the appearance of prespores and was completed prior to the appearance of mature spores. After $12 \mathrm{hr}$, mannan remained at a constant level of $8 \mu \mathrm{g} / 10^{8}$ spheroplasts. In the alkali-insoluble fraction, the accumulation of glucose and glucosamine showed little difference (Fig. 5B). The results show that the amount of insoluble carbohydrates rose slightly during spore formation. But, glucose rose again betwen 16 and $24 \mathrm{hr}$, increasing to twice the level present in the spore walls by $8 \mathrm{hr}$.

\section{DISCUSSION}

Carbohydrate metabolism during ascospore development in whole cells of Saccharomyces cerevisiae was reported by Kane and Roth.5) They could not rule out the possibility that the carbohydrate was derived from pre-existing material in the vegetative cells. Previously, we reported that comparison of the compositions of spore walls after sporulation in whole cells and spheroplasts of $S$. cerevisiae strain FE-1 did not show quantitative variations in the contents of lipid, carbohydrate and protein. ${ }^{13}$ ) Our results show that a system for synchronous sporulation in spheroplasts will be useful for solving the problem of ascus wall contamination.

The sporulated spheroplasts were fractionated into three fractions: extraspore material, spore-cytoplasm and spore walls. The major component of the extraspore fraction was glycogen. The glycogen was synthesized early in development and was degraded prior to spore formation (Fig. 3A), probably serving as a source of energy or carbon, or both, for spore formation. These results agree with other results. ${ }^{1,3 \sim 6)}$ The carbohydrates of the spore-cytoplasm fraction were fractionated into three components: trehalose, mannan and glycogen. As previously reported, ${ }^{14)}$ we found that carbohydrates (trehalose and glycogen) newly formed during developement were accumulated as sources of energy or carbon, or both, and were consumed during spore germination. It is necessary to determine whether the mannan in the spore-cytoplasm is a precursor of mannan or a source of energy. 
The spore wall carbohydrates were fractionated after extraction with alkali. The alkali-soluble fraction contained glucose and mannose. Since the sugars in the alkalisoluble fraction were detected in the void volume fractions on chromatography on Sephadex G-100 (data not shown), and were mainly obtained as trimethylated sugars on methylation analysis (Table I), it appeared that the sugars were released as polysaccharides on extraction. The mannan, as a minor component of the spore walls, could be almost completely extracted. The mannan synthesis was completed prior to spore maturation. About 60 to $80 \%$ of the glucose in the spore walls could be extracted between 8 and 24-hr incubation. However, the possibility remains that a considerable amount of glucan, synthesized during the first 8-hr incubation, becomes alkali-insoluble on covalent linking to the glucosamine polymer and so on. The newly synthesized polysaccharide in the soluble fraction was mainly $1,3-$ linked glucan during sporulation, as found on methylation analysis. During sporulation, other polysaccharides, perhaps 1,6-glucan and glycogen, may also be accumulated in the spore wall. The fine structure of the polysaccharide was not characterized, since further fractionation was not carried out. Chitin or a chitin-like polymer is a structural component of the outer layers of the spore wall. ${ }^{13,18,19)}$ The accumulation of the polymer began prior to the appearance of prespores and was completed within the period of spore formation. The results of chemical analyses and fluorescence microscopic observation suggested that the outermost surface of the chitin layer of the prespore wall might be covered with another substance(s), that prevents the chitin from binding with an added chitin-specific fluorescent dye during spore maturation. Recently, Briza et al. $^{20)}$ reported that dityrosine was a sporulation-specific component of the outermost osmophilic layers of the yeast ascospore wall. In the previous study, ${ }^{13)}$ we found that the outer layer of the spore wall was hydrolyzed only a little on chitinase digestion.

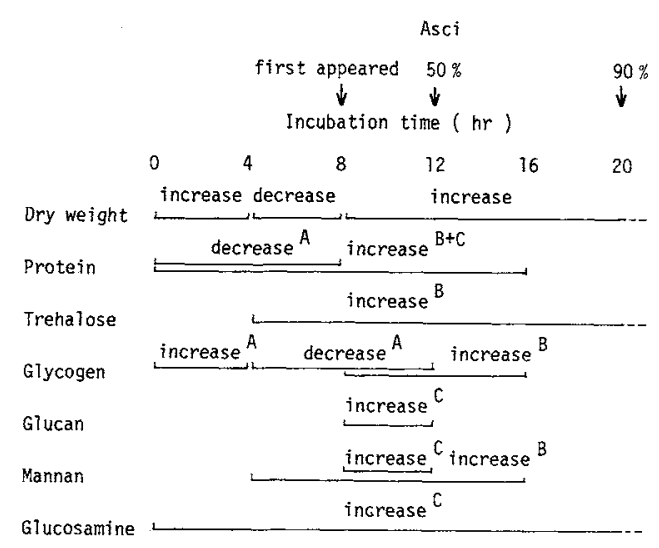

FlG. 6. Temporal Relationship of Events during Sporulation in Spheroplasts.

The durations of the periods of decreases and increases in the extraspore material (A), spore-cytoplasm (B) and spore walls (C) are indicated by the bars.

The possibility remains that dityrosine and glucosamine are parts of the same structure which cannot be hydrolyzed on the enzyme digestion.

Figure 6 shows a diagram of the timing of carbohydrate metabolism during sporulation. Glycogen in the extraspore material is synthesized early in developement and is completely degraded prior to spore formation. Trehalose, glycogen and mannan synthesized in the sporecytoplasm during sporulation are considered to be reserve or storage materials for spore germination. ${ }^{14)}$ Glucan, as a major component of the spore wall, is synthesized during spore maturation. The glucosamine polymer, as a component of the outer layers of the spore wall, is synthesized immediately after incubation in the sporulation medium.

Sporulation in spheroplasts may provide a convenient system for the investigation of the mechanism of synthesis of the spore wall. The enzyme-catalyzed degradation of glycogen and protein during sporulation in spheroplasts will be the subject of further work.

Acknowledgments. The authors express their sincere thanks to Professor Nobundo Sando of the Faculty of Science, Yamaguchi University, Yamaguchi, and Dr. Katsuji Ueki, Yamagata University, for the discussions and criticism. 


\section{REFERENCES}

1) R. D. Pontefract and J. J. Miller, Can. J. Microbiol., 8, 573 (1962).

2) R. Roth, J. Bacteriol., 101, 53 (1970).

3) P. Galzy, F. Vezinhet, A. Arnaud and A. ChassangDouillet, Metabolic modifications during sporulation in Saccharomyces cerevisiae Hansen, Fermentation Technology Today. Proc. 4th Int. Fermentation Symp., 1972, p. 863.

4) M. Tingle, A. J. S. Klar, S. A. Henry and H. O. Holvorson, Soc. Gen. Microbiol., 23, 209 (1973).

5) S. M. Kane and R. Roth. J. Bacteriol., 118, 8 (1974)

6) A. K. Hopper, P. T. Magee, S. K. Welch, M. Friedman and B. D. Hall, J. Bacteriol., 119, 619 (1974).

7) W. J. Colonna and P. T. Magee, J. Bacteriol., 134, 844 (1978).

8) W. A. Fonzi, M. Shanley and D. J. Opheim, J. Bacteriol., 137, 285 (1979).

9) M. J. Clancy, L. M. Smith and P. T. Magee, Mol. Cell Biol., 2, 171 (1982).

10) N. Sando, in "Growth and Differentiation in
Microorganisms," ed. by T. Ishikawa, Y. Maruyama and M. Matsumiya, University of Tokyo Press, Tokyo, 1977, pp. 151 163 .

11) K. Ueki, M. Abe, K. Tada and N. Sando, J. Gen. Microbiol., 129, 3619 (1983).

12) K. Ueki, Y. Shida and T. Tanji, J. Gen. Appl. Microbiol., 27, 1 (1981).

13) S. Katohda, K. Konno, Y. Sasaki, K. Suzuki and S. Sakamoto, Agric. Biol. Chem., 48, 895 (1984).

14) S. Katohda, M. Ito, K. Sasaki and M. Takahashi, Agric. Biol. Chem., 51, 2975 (1987).

15) S. Hakomori, J. Biochem., 55, 205 (1964).

16) S. Katohda, M. Tsukimaga, Y. Tobinai and T. Sato, Agric. Biol. Chem., 46, 1131 (1982).

17) M. Hayashibe and S. Katohda, J. Gen. Appl. Microbiol., 19, 23 (1973).

18) W. L. Whelan and C. E. Ballou, J. Bacteriol., 124, 1545 (1975).

19) N. Sando, T. Oguchi, M. Nagano and M. Osumi, $J$. Gen. Appl. Microbiol., 26, 403 (1980).

20) P. Briza, G. Winkler, H. Kalchhauser and $M$. Breitenbach, J. Biol. Chem., 261, 4288 (1986). 